\title{
Biblioteca de juegos serios para hacer más efectiva la enseñanza de Kanban acorde a objetivos de aprendizaje enfocados
}

\author{
Mirna Muñoz ${ }^{1}$ Miguel Ángel González Pacheco' ${ }^{1}$, José Guadalupe Hernández Reveles² \\ \{mirna.munoz, miguel.gonzalez\}@cimat.mx; pphdez@jag.ac \\ ${ }^{1}$ Centro de Investigación en Matemáticas- Unidad Zacatecas, Parque Quantum, Ciudad el Conocimiento \\ Avenida Lassec, Andador Galileo Galilei, Manzana, 3 Lote 7, 98160. Zacatecas, México. \\ ${ }^{2}$ Kueski. Circuito Coru.a 68, Fracc. Lomas de Galicia, Guadalupe, Zac
}

DOI: 10.17013/risti.41.1-16

\begin{abstract}
Resumen: En los últimos años el uso de Kanban se ha popularizado e incrementado en la industria del software. Sin embargo, la falta de experiencia y la difícil formación de los nuevos practicantes limitan su adopción dentro de las organizaciones. Una alternativa para resolver este problema, es hacer uso de juegos serios como herramienta en la capacitación del capital humano, ya que impulsan un cambio en su actitud, motivación y compromiso. Sin embargo, al existir muchos juegos serios con este propósito dificulta la elección del más adecuado, si no se cuenta con la debida experiencia. Este artículo presenta una biblioteca de juegos serios para enseñar Kanban desarrollada con el propósito de ayudar a los entrenadores a seleccionar el juego serio que cumpla con sus objetivos de enseñanza. La biblioteca fue validada por un grupo de expertos en Kanban. Los resultados destacan que la biblioteca ayuda a centralizar la información de los juegos serios y facilita la selección de juegos adecuados a los objetivos de aprendizaje establecidos a entrenadores poco experimentados.
\end{abstract}

Palabras-clave: juegos serios, Kanban, formación, biblioteca de juegos serios, ingeniería de software, mejora de procesos.

\section{Serious game library to make the teaching of Kanban more effective according to the learning objectives targeted}

Abstract: In recent years the use of Kanban has become popular and its use in the
software industry increased. However, the lack of experience and the difficult in the
training of new practitioners have limited its adoption within organizations. As an
alternative to solve this problem, is the use of serious games as a tool to training
human resources because they address a change in their attitude, motivation and
commitment. However, there are many serious games for this purpose that makes
difficult to choose the most appropriate game, if trainers do not have an adequate
experience. This paper presents a serious game library for teaching Kanban 
developed, which aims to help trainers to select the serious game that meets their teaching objectives. The library was validated by a set of Kanban experts. The results highlight that the library helps to centralize the serious games information, and facilitates the selection of the games according to the established learning objectives for inexperienced trainers.

Keywords: serious games, Kanban, training, serios game library, software engineering, process improvement.

\section{Introducción}

El desarrollo de software enmarca un conjunto de actividades llevadas acabo mediante el proceso de creación, diseño, implementación y soporte de software (IBM, 2014). La ingeniería de software es el área dentro de las Ciencias de la Computación encargada de mejorar este proceso para hacerlo más rápido y con mayor calidad, para lograr este propósito, en esta área se generar una gran gama de metodologías, frameworks, herramientas y métodos.

En este contexto, en los últimos años, en un esfuerzo por hacer de proceso de desarrollo de software más rápido, se ha incrementado el uso de métodos ágiles para el desarrollo de software (State of Agile, 2019). Uno que se ha destacado es Kanban, que es una herramienta que permite gestionar las actividades del proceso de desarrollo, logrando un cambio en la forma en la que se producen y se entregan los productos y servicios (Anderson, 2010).

Sin embargo, no hay muchos expertos en Kanban, por lo que resulta difícil aumentar el número de profesionistas del desarrollo de software que lo usen, lo que ocasiona una limitada adopción dentro de las organizaciones (Ahmad et al., 2013).

Como una solución a esta problemática y con el fin de incrementar el número de practicantes de Kanban, se ha recurrido al aprendizaje basado en el juego (Game Based Learning, GBL) ya que ha demostrado que es posible aprender y evaluar el conocimiento adquirido mientras se fomenta un cambio positivo en la actitud, motivación y compromiso de los participantes (Hernández \& Cadavid, 2019). Por lo que los juegos serios han sido utilizados en la capacitación en diferentes áreas como: política, defensa, salud, sector educativo y las organizaciones (Hernández \& Cadavid, 2019).

Para el caso de formación en Kanban, aun cuando ya se utilizan juegos serios como parte de sus capacitaciones, debido a la gran cantidad de juegos, la poca o nula documentación dispersa de los mismos, así como la falta de experiencia por parte de los entrenadores junior, dificulta la selección de los juegos más adecuados acorde a los oebjtivos de aprendizaje pretentidos.

Con base en la problemática mencionada, este artículo presenta una biblioteca de juegos serios para enseñar Kanban cuyo objetivo es servir de apoyo a entrenadores, principalmente a los junior, que buscan reforzar la enseñanza de los conceptos básicos de Kanban de una forma más eficiente, y de acuerdo al perfil de los participantesy necesidades y objetivos de aprendizaje establecidos.

Después de la introducción, este artículo se estructura como sigue: la sección 2, se presenta una breve definición de Kanban y juegos serios; la sección 3, muestran los 
trabajos relacionados; la sección 4, presenta la biblioteca de juegos serios para enseñar Kanban; la sección 5, muestra la validación de la biblioteca; y la sección 6, presenta las conclusiones.

\section{Definiciones de Kanban y juegos serios}

\subsection{Kanban}

Kanban es una palabra japonesa que significa "señal" o "tarjeta", y fue originada y aplicada por primera vez en Toyota, para la industria de manufactura (Ohno, 1988). El objetivo principal de Kanban es entregar valor de forma rápida, con el uso adecuado de recursos para eliminar el desperdicio y así lograr una mayor productividad y calidad en los productos (Liker et al., 2008; Magee, 2008).

Anderson (2010), se percató de que Kanban podría aplicarse en cualquier otro tipo de industria, no sólo la automotriz, y lo adapta al desarrollo de software. Por lo tanto, hoy en día, Kanban es explicado de mejor manera a través de sus principios y prácticas fundamentales definidas por Anderson \& Carmichael en (Anderson \& Carmichael, 2016) y Burrows \& Hohmann en (Burrows \& Hohmann, 2014).

Los principios de Kanban se dividen en dos grupos:

- Principios de gestión del cambio. Kanban reconoce que los individuos que conforman a las organizaciones son resistentes al cambio, por lo tanto, recomienda estos tres principios para reducción de resistencia al cambio y la obtención del compromiso de los individuos para la mejora: 1) partir de lo que ya haces, 2) entender el proceso actual, y 3) respetar los roles, responsabilidades y los títulos actuales.

- Principios de entrega de servicios. Las organizaciones forman ecosistemas de servicios que dependen de unos con otros, Kanban recomienda tres principios que se aplican a todos esos servicios: 1) conocer y centrarse en las necesidades de los clientes, 2) permitir que los individuos sean auto-organizados en sus labores, y 3) experimentar cambios de mejora de las políticas para favorecer los resultados con los clientes y el negocio.

Las prácticas fundamentales de Kanban se listan a continuación:

1. Visualizar: se refiere a tener tangible el trabajo y el proceso que conlleva. A menudo se utilizan tableros electrónicos o en otros medios, y estos varían entre sistemas Kanban ya que depende de cómo serán usados.

2. Limitar el trabajo en proceso: se refiere a determinar y comprometerse a límites de trabajo en proceso, actividad clave de un sistema de trabajo pull, donde los objetos no empiezan hasta que el trabajo actual sea completado o abortado.

3. Gestionar el flujo: se refiere a que el proceso se debe enfocar en maximizar la entrega de valor, reducir los tiempos de entrega e intentar ser predecible.

4. Hacer las políticas explícitas: se refiere a que las políticas deben ser pocas, entendibles, estar bien definidas y al alcance de todos para que puedan ser aplicadas, sin olvidar que pueden ser cambiadas con el fin de mejorar. 
5. Implementar ciclos de retroalimentación: se refiere a realizar actividades de retroalimentación para lograr la mejora del proceso, enfocando en alineación estratégica, coordinación operativa, gestión del riesgo, mejora del servicio, reabastecimiento, flujo y entregas a los clientes.

6. Mejorar colaborativamente, evolucionar experimentando: se refiere al uso de un paradigma Lean, parte de lo que ya haces y busca una mejora continua por medio de la experimentación.

\subsection{Juegos serios}

El termino se popularizó en la iniciativa de juegos serios de 2002 que tenía como objetivo enfocar en el uso de los juegos para explorar los desafíos de gestión y liderazgo que enfrentaba el sector público (Susi et al., 2015).

Entre las definiciones de juegos serios Michael \& Chen (2006), mencionan que los juegos son aquellos que los mismos objetivos que el entrenamiento educativo, pero que no están delimitados solo al propósito de memorizar o enfatizar en los hechos, siendo lo más importante, que los participantes aprendan y se diviertan.

Zyda (2005, p. 26) por otra parte, describe a los juegos serios como retos mentales que se juegan a través de una computadora con reglas previamente establecidas, por lo que éstos son utilizados dentro del sector salud, educativo, política, el gobierno y empresas como herramienta de capacitación.

\section{Trabajos relacionados}

Esta sección presenta dos trabajos relacionados con la investigación, los cuales fueron seleccionados porque presentan estudios de caso donde se enseña Kanban a través de juegos serios.

La relación que hay entre estos trabajos y el que se realizó en esta investigación, es que utilizan juegos serios para enseñar el método Kanban a un grupo de interés, los estudios buscan evaluar un juego serio para enseñar Kanban y analizan si se alcanzan los objetivos de aprendizaje. Sin embargo, cabe resaltar que la biblioteca de juegos serios tiene como objetivo recolectar juegos serios para enseñar Kanban, analizar sus características y evaluar su eficacia para ofrecer herramientas a los entrenadores para enseñar el método.

López y Petrillo (Lopes \& Petrillo, 2017) presentan el juego serio SimKan que utiliza una simulación estocástica para introducir aspectos aleatorios en el juego, permitiendo una experiencia cuasi verdadera en Kanban implementada en un lapso corto de tiempo y utilizando simples herramientas. SimKan para reproducir escenarios de simulación de proyectos de software para entrenar en el uso de Kanban. Simkan permite a los participantes experimentar en poco tiempo y a bajo costo las prácticas de Kanban. Por lo tanto, es posible recoger métricas, llevar a cabo debates, y adquirir experiencia sin el riesgo y el tiempo necesarios de un proyecto real, con sus características y compromisos establecidos. Los resultados muestran que SimKan es adecuado para entrenar equipos Kanban brindando resultados positivos a corto plazo y bajo costo. 
Heikkilä, Paasivaara y Lassenius (Heikkilä et al., 2016) presentan un juego de mesa colaborativo para enseñar Kanban en un curso de gestión de proyectos de software en la Universidad Aalto. El objetivo del juego es medir el alcance de los objetivos de aprendizaje de la clase y estudiar la percepción de los estudiantes sobre el juego. Se recolectaron datos de dos clases en 2014 y 2015. Reunieron datos cuantitativos con cuestionarios que fueron analizados de forma descriptiva y estadística. Los estudiantes percibieron que habían aprendido bastante del juego y evaluaron su experiencia de forma muy positiva. Sin embargo, los resultados cualitativos y aprendizaje medido indicaron que los objetivos de aprendizaje se alcanzaron de forma parcial.

\section{Biblioteca de juegos serios para enseñar Kanban}

Para el desarrollo de la biblioteca se realizaron cuatro actividades que se describen a continuación:

\subsection{Identificar juegos serios para enseñar Kanban}

Se identificaron doce juegos serios para enseñar Kanban, cada juego fue analizado, para extraer sus características más importantes, consideradas de utilidad para la selección del juego de acuerdo a objetivos de aprendizaje. Los juegos serios que fueron seleccionados en su mayoría son de libre acceso. La Tabla 1 muestra los doce juegos seleccionados para ser incluidos en la biblioteca, en cada uno se lista el nivel de cobertura sobre los siete objetivos de aprendizaje de Kanban: (1) entender los conceptos básicos de Kanban; (2) entender cómo funciona un sistema Kanban; (3) aprender los 3 principios de gestión de cambio; (4) aprender los principios y prácticas fundamentales Kanban; (5) experimentar los beneficios de limitar el trabajo en proceso para mejorar el flujo de trabajo; (6) entender cómo implementar reuniones Kanban en torno al trabajo, y (7) entender las métricas principales de Kanban.

\begin{tabular}{|c|c|c|}
\hline ID & Nombre & Objetivos \\
\hline 1 & $\begin{array}{l}\text { GetKanban (v5.o) } \\
\text { (GetKanban, 2020) }\end{array}$ & $\begin{array}{l}\text { TC: (1) entender los conceptos básicos de Kanban; (2) entender cómo } \\
\text { funciona un sistema Kanban; (5) experimentar los beneficios de limitar } \\
\text { el trabajo en proceso para mejorar el flujo de trabajo; (6) entender cómo } \\
\text { implementar reuniones Kanban en torno al trabajo; (7) entender las métricas } \\
\text { principales de Kanban. } \\
\text { PC: (4) aprender los principios y prácticas fundamentales de Kanban }\end{array}$ \\
\hline 2 & $\begin{array}{l}\text { GetKanban (v2.o) } \\
\text { (GetKanban, 2020) }\end{array}$ & $\begin{array}{l}\text { Edición gratuita de GetKanban, con la particularidad de que puede ser } \\
\text { modificado para alcanzar otros objetivos de aprendizaje. } \\
\text { TC: (1) entender los conceptos básicos de Kanban; (2) entender cómo } \\
\text { funciona un sistema Kanban; (5) experimentar los beneficios de limitar } \\
\text { el trabajo en proceso para mejorar el flujo de trabajo; (6) entender cómo } \\
\text { implementar reuniones Kanban en torno al trabajo; (7) entender las métricas } \\
\text { principales de Kanban. } \\
\text { PC: (4) aprender los principios y prácticas fundamentales de Kanban }\end{array}$ \\
\hline 3 & $\begin{array}{l}\text { Ask Kanban } \\
\text { (Wovchko, 2016) }\end{array}$ & TC: (6) entender cómo implementar reuniones Kanban en torno al trabajo. \\
\hline
\end{tabular}




\begin{tabular}{|c|c|c|}
\hline ID & Nombre & Objetivos \\
\hline 4 & $\begin{array}{l}\text { Kanban Pizza Game } \\
\text { (Agile42, 2020) }\end{array}$ & $\begin{array}{l}\text { TC: (1) entender los conceptos básicos de Kanban; (2) entender cómo } \\
\text { funciona un sistema Kanban; (4) aprender los principios y prácticas } \\
\text { fundamentales de Kanban; (5) experimentar los beneficios de limitar el } \\
\text { trabajo en proceso para mejorar el flujo de trabajo. } \\
\text { PC: (6) entender cómo implementar reuniones Kanban en torno al trabajo. }\end{array}$ \\
\hline 5 & $\begin{array}{l}\text { Decoration Kanban } \\
\text { (Tastycupcakes, } \\
\text { 2020) }\end{array}$ & $\begin{array}{l}\text { TC: (1) entender los conceptos básicos de Kanban; (5) experimentar los } \\
\text { beneficios de limitar el trabajo en proceso para mejorar el flujo de trabajo. } \\
\text { PC: (2) entender cómo funciona un sistema Kanban; (4) aprender los } \\
\text { principios y prácticas fundamentales de Kanban. }\end{array}$ \\
\hline 6 & $\begin{array}{l}\text { The Penny Game } \\
\text { (Netmind, 2019) }\end{array}$ & $\begin{array}{l}\text { TC: (5) experimentar los beneficios de limitar el trabajo en proceso para } \\
\text { mejorar el flujo de trabajo. }\end{array}$ \\
\hline 7 & $\begin{array}{l}\text { Sudokuban } \\
\text { (Unbound DNA, } \\
\text { 2014) }\end{array}$ & $\begin{array}{l}\text { TC: (2) entender cómo funciona un sistema Kanban; (5) experimentar los } \\
\text { beneficios de limitar el trabajo en proceso para mejorar el flujo de trabajo. } \\
\text { PC: (1) entender cómo funciona un sistema Kanban; (4) aprender los } \\
\text { principios y prácticas fundamentales de Kanban. }\end{array}$ \\
\hline 8 & $\begin{array}{l}\text { Kanban Tetris } \\
\text { (Tastycupcakes, } \\
\text { 2020) }\end{array}$ & $\begin{array}{l}\text { TC: (2) entender cómo funciona un sistema Kanban; (5) experimentar los } \\
\text { beneficios de limitar el trabajo en proceso para mejorar el flujo de trabajo. } \\
\text { PC: (1) entender cómo funciona un sistema Kanban; }\end{array}$ \\
\hline 9 & $\begin{array}{l}\text { Kanban 1s Game } \\
\text { (Jon Jagger, s.f.) }\end{array}$ & $\begin{array}{l}\text { TC: (5) experimentar los beneficios de limitar el trabajo en proceso para } \\
\text { mejorar el flujo de trabajo. }\end{array}$ \\
\hline 10 & $\begin{array}{l}\text { A Dice Game } \\
\text { (LitheSpeed, 2020) }\end{array}$ & $\begin{array}{l}\text { TC: (5) experimentar los beneficios de limitar el trabajo en proceso para } \\
\text { mejorar el flujo de trabajo. }\end{array}$ \\
\hline 11 & $\begin{array}{l}\text { Featureban (v3.0) } \\
\text { (Agendashift, 2015) }\end{array}$ & $\begin{array}{l}\text { TC: (1) entender los conceptos básicos de Kanban; (2) entender cómo } \\
\text { funciona un sistema Kanban; (4) aprender los principios y prácticas } \\
\text { fundamentales de Kanban; (5) experimentar los beneficios de limitar el } \\
\text { trabajo en proceso para mejorar el flujo de trabajo; (7) entender las métricas } \\
\text { principales de Kanban. } \\
\text { PC: (6) entender cómo implementar reuniones Kanban en torno al trabajo. }\end{array}$ \\
\hline 12 & $\begin{array}{l}\text { Juego de los barcos } \\
\text { de papel } \\
\text { (Klaus Leopold, s.f.) }\end{array}$ & $\begin{array}{l}\text { TC:(1) entender los conceptos básicos de Kanban; (2) entender cómo funciona } \\
\text { un sistema Kanban; (5) experimentar los beneficios de limitar el trabajo en } \\
\text { proceso para mejorar el flujo de trabajo. } \\
\text { PC: (4) aprender los principios y prácticas fundamentales de Kanban. }\end{array}$ \\
\hline
\end{tabular}

TC: totalmente cubierto; PC: parcialmente cubierto

Tabla 1 - Juegos seleccionados para enseñar Kanban

\subsection{Probar y evaluar la eficacia de los juegos serios}

Para probar y evaluar la eficiencia de los juegos, se seleccionó una muestra de dos de los juegos (Featureban v3 y el juego de los barcos de papel), y se implementaron en talleres realizados en tres ciudades de México: Aguascalientes, Pachuca y Zacatecas. Los juegos serios se utilizaron como herramienta de apoyo para enseñar Kanban en un ambiente real.

Los juegos serios fueron evaluados siguiendo la propuesta de Ghannem (2014), por lo que se enfocó en la usabilidad y el cumplimiento de sus objetivos de aprendizaje, así como la motivación e inmersión de los participantes. Para realizar la medición, se elaboró un cuestionario dividido en dos partes. La primera parte, implementada al iniciar el taller, tuvo como objetivo conocer los datos demográficos, experiencia previa en Kanban y 
obtener un punto de referencia de los conocimientos de los conceptos básicos de Kanban de los participantes. La segunda parte, implementada posterior a finalizar el juego, tuvo como objetivo medir el conocimiento adquirido de los conceptos básicos de Kanban, así como la percepción de los jugadores hacia el juego, su motivación e inmersión al momento de jugar.

Además, se utilizó una escala de Likert de 10 valores para evaluar el conocimiento de los participantes para cada concepto de Kanban. Las encuestas fueron utilizadas en los tres talleres respectivamente, sin embargo, para el último taller se modificó la forma en la que se evaluaron los conceptos básicos de Kanban, utilizando opciones múltiples con respuestas correctas que otorgaban puntos positivos, y respuestas incorrectas que otorgaban puntos negativos, de esta forma se calculaba su valoración entre o y 10. La Figura 1 muestra los resultados del promedio para cada concepto de Kanban de las encuesta previa y posterior de cada taller.

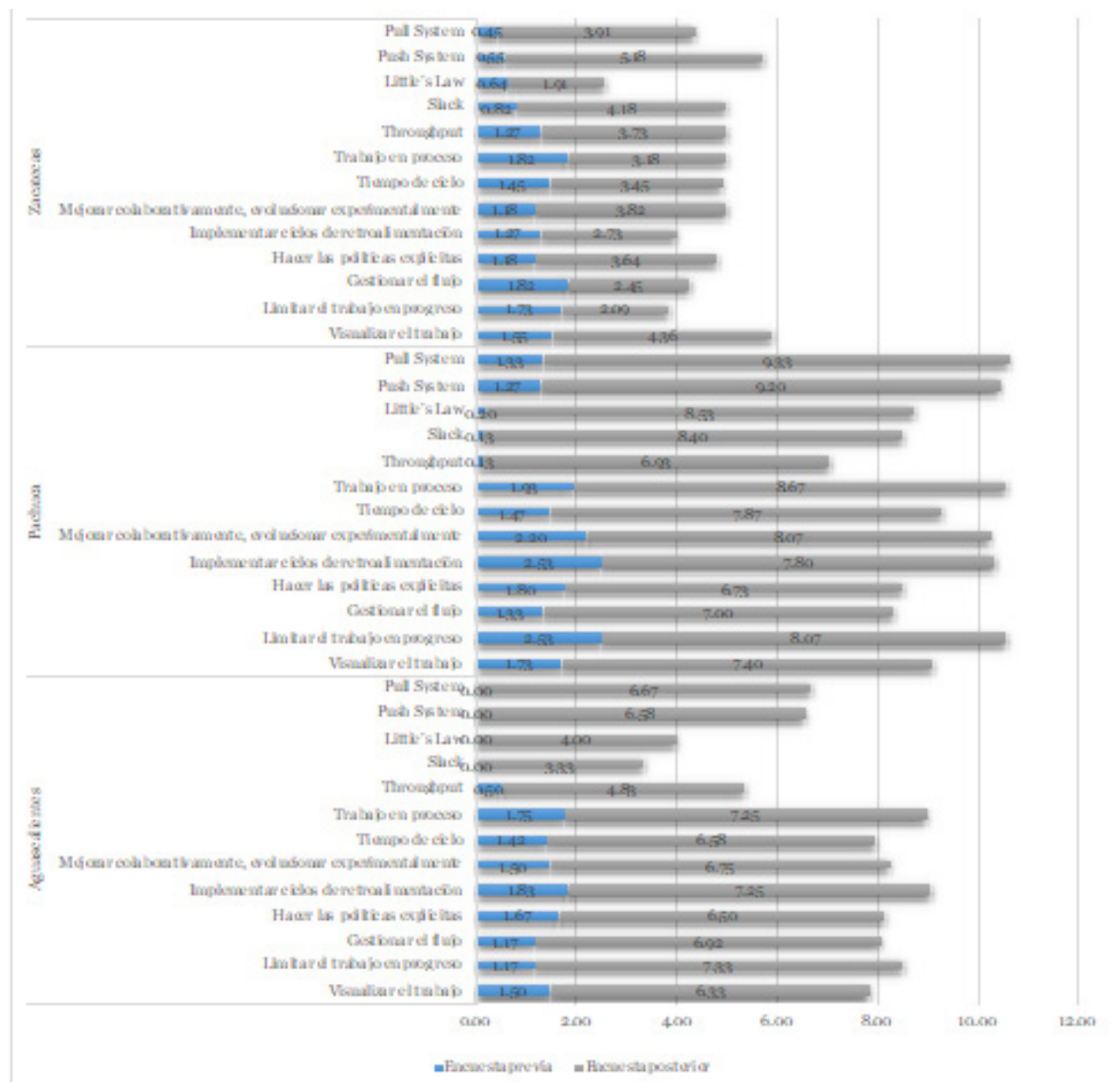

Figura 1 - Resultados del promedio de conocimiento de los conceptos de Kanban de los talleres 
Como se observa en la gráfica de la Figura 1, en Aguascalientes los conceptos básicos que obtuvieron los mejores resultados fueron "Limitar el trabajo en proceso" e "Implementar ciclos de retroalimentación” con 7.33 y 7.25 respectivamente. Las principales métricas tuvieron una mejora significativa una vez que se explicaron con la ayuda de los juegos serios. Los conceptos sobre los sistemas de trabajo también obtuvieron resultados significativos, puesto que pasaron de un promedio de o a superiores a 6.

En el caso de Pachuca, los conceptos básicos de Kanban que obtuvieron mejores resultados fueron "Limitar el trabajo en proceso" y "Mejorar colaborativamente, evolucionar experimentado" cuyos promedios son de 8. Al igual que en Aguascalientes, las principales métricas también obtuvieron mejoras significativas al pasar de promedios inferiores a 2 a promedios superiores a 6. Lo mismo pasó con los conceptos sobre los sistemas de trabajo que obtuvieron promedios superiores a 9, así mismo, los conceptos de "Slack" y "Little's Law" obtuvieron promedios superiores a 8 debido a que el instructor constantemente está mejorando su curso.

En Zacatecas la diferencia es visible, y esto se debe al cambio en la forma de evaluar los conocimientos obtenidos. Aun así, se puede ver que también hubo mejora en todos los conceptos. Los conceptos básicos que obtuvieron los mejores resultados son "Visualizar el trabajo", "Hacer las políticas explícitas" y "Mejorar colaborativamente, evolucionar experimentado" con promedios que van desde 1.55 a 4.36, 1.18 a 3.64 y 1.18 a 3.82 respectivamente. Las principales métricas obtuvieron promedios superiores a 3, los conceptos de "Slack" y "Little's Law" pasaron de promedios inferiores a 1 a promedios de 4.18 para "Slack" y 1.91 para "Little's Law". Finalmente, los sistemas de trabajo pasaron de promedios inferiores a 1 , a promedios de 5.18 para el sistema push y 3.91 para el sistema pull.

Estos resultados concuerdan con los objetivos de aprendizaje que persiguen los juegos serios aplicados ya que los participantes experimentaron los beneficios de limitar el trabajo en proceso con el uso de un tablero Kanban, así como simular ambos sistemas de trabajo (sistema push y sistema pull) para identificar sus diferencias.

\subsection{Desarrollo de la biblioteca}

La biblioteca se desarrolló siguiendo un proceso de cuatro etapas principales, en cada una se realizaron diferentes actividades que se describen a continuación en la Tabla 2.

\begin{tabular}{llll}
\hline Etapa & Descripción & Actividades & Productos generados \\
\hline Análisis de & Entendimiento y definición & Establecer los requisitos del & Documento de visión y \\
requisitos & de los requisitos del & proyecto & alcance \\
& proyecto y establecimiento & Definir los objetivos del & Documento de requisitos \\
& de los planes de desarrollo & proyecto & arquitecturales \\
& de la biblioteca & Establecer las métricas & \\
& de éxito, características & \\
& principales, riesgos, & \\
& asunciones y dependencias, & \\
& alcance y limitaciones & \\
\hline
\end{tabular}




\begin{tabular}{|c|c|c|c|}
\hline Etapa & Descripción & Actividades & Productos generados \\
\hline \multirow[t]{3}{*}{$\begin{array}{l}\text { Diseño de alto y } \\
\text { bajo nivel }\end{array}$} & \multirow{3}{*}{$\begin{array}{l}\text { Elaboración de los } \\
\text { diagramas que describen el } \\
\text { proyecto de software tanto } \\
\text { del funcionamiento general } \\
\text { y detallado }\end{array}$} & $\begin{array}{l}\text { Realizar la selección de la } \\
\text { tecnología para el desarrollo } \\
\text { de software }\end{array}$ & \multirow[t]{3}{*}{ Documento de diseño } \\
\hline & & $\begin{array}{l}\text { Elaborar un diagrama de } \\
\text { contexto y de contenedores } \\
\text { que describen la estructura } \\
\text { general del software }\end{array}$ & \\
\hline & & $\begin{array}{l}\text { Elaborar diagramas de casos } \\
\text { de uso y de flujo }\end{array}$ & \\
\hline \multirow{2}{*}{$\begin{array}{l}\text { Desarrollo y } \\
\text { pruebas }\end{array}$} & \multirow{2}{*}{$\begin{array}{l}\text { Codificar el producto de } \\
\text { software y realizar las } \\
\text { pruebas a la par de la } \\
\text { codificación }\end{array}$} & Codificar & \multirow[t]{2}{*}{ Código fuente } \\
\hline & & Probar & \\
\hline \multirow[t]{3}{*}{$\begin{array}{l}\text { Lanzamiento o } \\
\text { despliegue }\end{array}$} & \multirow{3}{*}{$\begin{array}{l}\text { Lanzar a producción el } \\
\text { producto de software } \\
\text { para que los entrenadores } \\
\text { puedan acceder a la } \\
\text { plataforma }\end{array}$} & $\begin{array}{l}\text { Preparar el proyecto para } \\
\text { su lanzamiento en Heroku } \\
\text { (Salesforce, 2020) }\end{array}$ & \multirow[t]{3}{*}{ Biblioteca en producción } \\
\hline & & $\begin{array}{l}\text { Lanzar la biblioteca a } \\
\text { producción }\end{array}$ & \\
\hline & & Realizar pruebas & \\
\hline
\end{tabular}

Tabla 2 - Proceso de desarrollo de la biblioteca

\subsection{Lanzamiento o despliegue de la interfaz de la biblioteca}

La biblioteca puede ser accedida desde el siguiente enlace https://bibliotecajuegosserios. herokuapp.com. A continuación se describen brevemente las tres partes principales que la componen:

La página principal (Figura 2), esta sección sirve para describir brevemente el contexto y motivaciones que llevaron a la realización de este trabajo, la experiencia obtenida al participar en los talleres, las partes que conforman la página web, la definición de los conceptos utilizados y sobre las personas que ayudarán en su desarrollo.

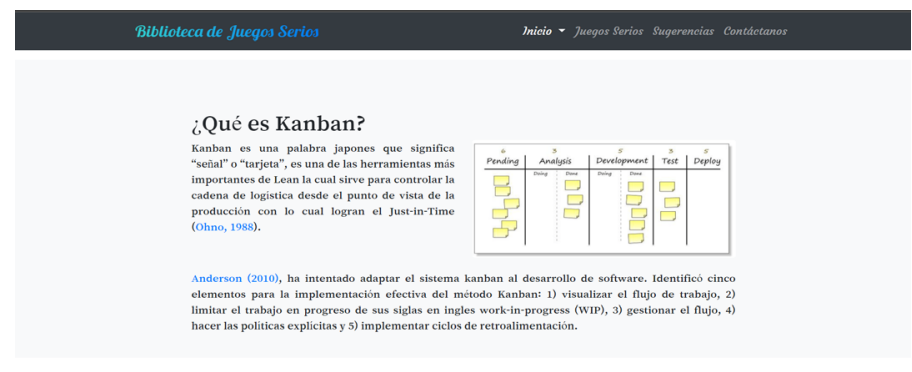

Figura 2 - Página principal de la biblioteca

La sección de la colección de los doce juegos serios donde es posible visualizar algunas de sus características (Figura 3). Así mismo, es posible visualizar todas las características de 
cada juego ingresando a los detalles. Para cada juego se describe cómo se implementan los principios, prácticas y métricas principales de Kanban, así como los objetivos de aprendizaje que cubre.

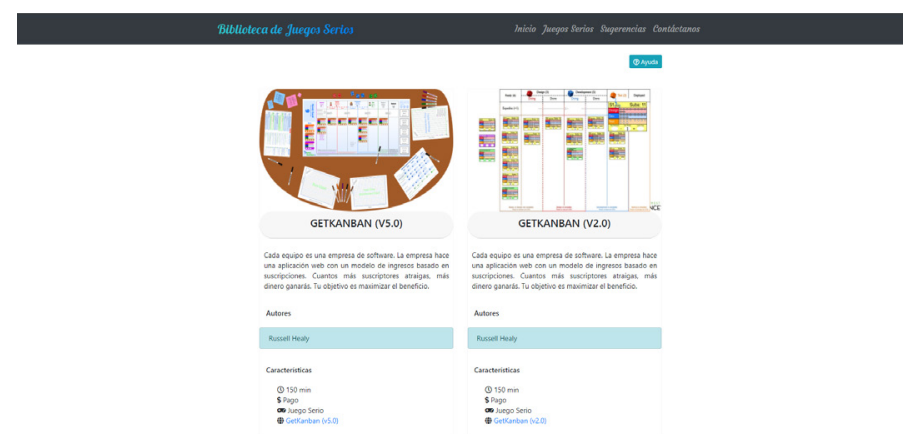

Figura 3- Colección de juegos serios

La sección de sugerencias (Figura 4), la cual tiene como objetivo brindar recomendaciones sobre objetivos de aprendizaje, juegos serios y consejos complementarios.

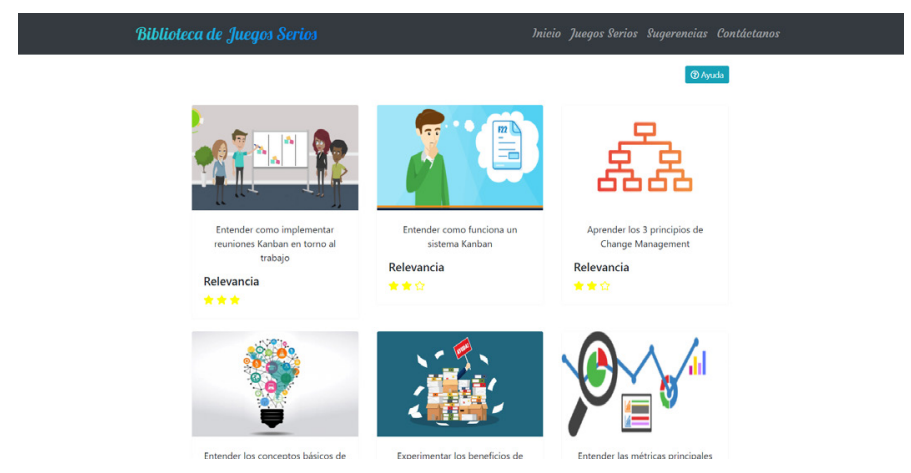

Figura 4 - Sugerencias complementarias

\section{Validación de la biblioteca}

Para validar la biblioteca se utilizó el método empírico de juicio de expertos, el proceso y las actividades que se realizaron se describe a continuación:

1. Presentación de la propuesta y definición de las características a evaluar: se redactó un documento donde se proporciona el contexto del problema, se explica la solución que se propone, y se muestra la propuesta que se desarrolló de la cual se desea obtener su opinión de expertos. Las características a evaluar se muestran en la Tabla 3. 


\begin{tabular}{ll}
\hline Característica & Descripción \\
\hline Colección de juegos serios & $\begin{array}{l}\text { Es una lista de juegos serios para enseñar Kanban descritos y } \\
\text { caracterizados. }\end{array}$ \\
\hline $\begin{array}{l}\text { Caracterización de los } \\
\text { juegos serios }\end{array}$ & $\begin{array}{l}\text { La caracterización se refiere a las secciones que presentan cada juego de } \\
\text { forma detallada, por ejemplo, autores, duración, licencia, tipo de actividad, } \\
\text { complejidad, dificultad, idioma, prerrequisitos, cantidad de jugadores, etc. }\end{array}$ \\
\hline $\begin{array}{l}\text { Cobertura de objetivos de } \\
\text { aprendizaje }\end{array}$ & $\begin{array}{l}\text { Los objetivos de aprendizaje mencionados en esta sección, están alineados } \\
\text { a la primera certificación de Kanban (Team Kanban PractitionerÒ) de la } \\
\text { Kanban University. En esa sección se describe si el juego serio cubre total, } \\
\text { parcial o no cubre dicho objetivo. }\end{array}$ \\
\hline $\begin{array}{l}\text { Principios, prácticas y } \\
\text { métricas fundamentales de } \\
\text { Kanban }\end{array}$ & $\begin{array}{l}\text { Estas secciones describen cómo cada juego serio implementa o no cada } \\
\text { principio y/o práctica de Kanban, así como la enseñanza de las principales } \\
\text { métricas. }\end{array}$ \\
\hline Sugerencias & $\begin{array}{l}\text { Son sugerencias sonbre juegos serios, objetivos de aprendizaje, y consejos } \\
\text { para los diferentes perfiles de participantes, que pueden servir para obtener } \\
\text { mejores resultados. }\end{array}$ \\
\hline
\end{tabular}

Tabla 3 - Características a evaluar

2. Definición de los expertos para su selección: se definieron dos perfiles y criterios que se deben cumplir para considerar a un individuo como experto. Para la validación de esta biblioteca se contó con la participación de 15 expertos con los perfiles y criterios mostrados en la Tabla 4.

\begin{tabular}{|c|c|c|c|}
\hline Perfil & Formación & Entorno laboral & Experiencia \\
\hline $\begin{array}{l}\text { Entrenadores } \\
\text { Kanban }\end{array}$ & $\begin{array}{l}\text { Contar al menos } \\
\text { con un certificado } \\
\text { Accredited Kanban } \\
\text { TrainerÒ (AKT) por la } \\
\text { Kanban University. }\end{array}$ & $\begin{array}{l}\text { Se dedica a dar cursos y/o } \\
\text { talleres de Kanban. } \\
\text { Da consultoría a las } \\
\text { organizaciones acerca de la } \\
\text { implementación de Kanban. }\end{array}$ & $\begin{array}{l}\text { Que tengan } \\
\text { conocimientos en } \\
\text { los materiales de } \\
\text { capacitación de KMP. } \\
\text { Tener por lo menos } 1 \text { año } \\
\text { de experiencia en el uso } \\
\text { del Método Kanban. } \\
\text { Haber impartido por } \\
\text { lo menos } 3 \text { cursos y/o } \\
\text { talleres de Kanban. }\end{array}$ \\
\hline $\begin{array}{l}\text { Profesores con } \\
\text { experiencia en } \\
\text { Kanban }\end{array}$ & $\begin{array}{l}\text { Haber asistido a } \\
\text { un taller o curso de } \\
\text { Kanban al mismo nivel } \\
\text { que la certificación } \\
\text { Kanban System Design } \\
\text { KMP1. }\end{array}$ & $\begin{array}{l}\text { Ser docente de una universidad } \\
\text { de carreras afines a las } \\
\text { Tecnologías de la Información } \\
\text { o Ciencias de la Computación } \\
\text { donde imparte Kanban como } \\
\text { curso o dentro de alguna } \\
\text { materia. } \\
\text { Es encargado de la capacitación } \\
\text { de una organización donde } \\
\text { imparte Kanban }\end{array}$ & $\begin{array}{l}\text { Conoce los conceptos } \\
\text { básicos del Método } \\
\text { Kanban, así como sus } \\
\text { prácticas, principios y } \\
\text { métricas fundamentales. } \\
\text { Tener experiencia en el } \\
\text { uso de Kanban, por lo } \\
\text { menos a nivel personal. }\end{array}$ \\
\hline
\end{tabular}

Tabla 4 - Perfiles y criterios de expertos 
3. Elaboración del instrumento de recolección de la información: se desarrolló un cuestionario para evaluar la utilidad, presentación y facilidad de uso de la biblioteca. Este instrumento fue elaborado utilizando Google Forms porque facilita la recolección y análisis de los datos. La Tabla 5 muestra las preguntas del instrumento de evaluación. La escala utilizada es de 1 a 5 como a continuación se describe: (1) Totalmente en desacuerdo; (2) En desacuerdo; (3) Ni en desacuerdo ni de acuerdo; (4) De acuerdo; (5) Totalmente de acuerdo.

\begin{tabular}{|c|c|}
\hline & Pregunta \\
\hline 1 & ¿Considera que tener una colección de juegos serios para enseñar Kanban es de utilidad para usted? \\
\hline 2 & $\begin{array}{l}\text { ¿Considera que las características de los juegos serios que se presentan en la biblioteca ayudan a la } \\
\text { elección del juego serio para enseñar Kanban? }\end{array}$ \\
\hline 3 & $\begin{array}{l}\text { ¿Considera que conocer la cobertura de los objetivos de aprendizaje para enseñar Kanban le ayuda } \\
\text { a elegir el juego serio que más se adecue a sus necesidades de enseñanza? }\end{array}$ \\
\hline 4 & $\begin{array}{l}\text { ¿Conocer el cómo cada juego serio implementa las prácticas, principios y métricas fundamentales } \\
\text { de Kanban le ayuda a elegir el juego serio para enseñar Kanban? }\end{array}$ \\
\hline 5 & $\begin{array}{l}\text { ¿Consultar sugerencias de acuerdo al perfil de los participantes y su experiencia en Kanban le } \\
\text { resulta útil? }\end{array}$ \\
\hline 6 & ¿Fue fácil acceder a la colección de juegos serios? \\
\hline 7 & ¿La forma en que se presentan las características de los juego serios le resulta adecuada? \\
\hline 8 & ¿Fue fácil consultar las sugerencias de acuerdo al perfil de los participantes? \\
\hline 9 & ¿La forma en que se presentan las sugerencias le facilita su comprensión? \\
\hline 10 & ¿Cuáles serían sus sugerencias de mejora? \\
\hline
\end{tabular}

Tabla 5 - Preguntas del instrumento de medición

4. Recolección de las respuestas de los expertos: se preparó un paquete con el documento de presentación de la propuesta, la definición de los perfiles de expertos y un enlace a la encuesta. Este paquete fue enviado por correo electrónico a los sujetos que cumplían con los criterios.

5. Análisis e interpretación de los datos: una vez terminado de recolectar las respuestas, se llevó acabo el análisis e interpretación de los datos.

\subsection{Análisis de resultados}

En esta sección se muestran los resultados obtenidos de las respuestas de los expertos a cada una de las preguntas del instrumento de medición.

Las preguntas de la 1 a la 5 evalúan la utilidad de la biblioteca, las pregunta 6 y 8 evalúan la facilidad de uso, las preguntas 7 y 9 evalúan la presentación y la pregunta 10 es un espacio abierto para que los expertos dejarán sus comentarios de mejora.

La Figura 5 muestra los resultados de las preguntas de la 1 a la 5 . 


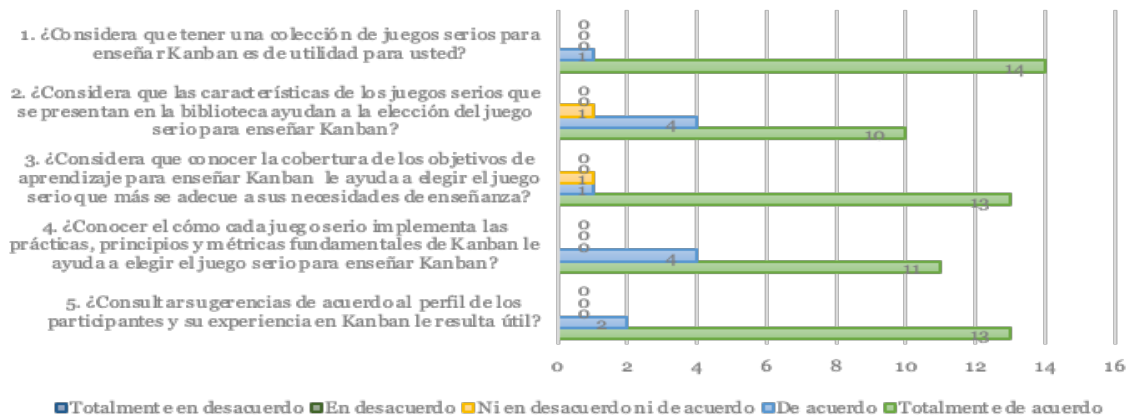

Figura 5 - Evaluación de la utilidad de la biblioteca

Como se oberva en la figura, los expertos están de acuerdo en que todas las características de la biblioteca son de utilidad para los entrenadores Kanban.

La Figura 6 muestra los resultados a las preguntas sobre la facilidad de uso de las características de la biblioteca.

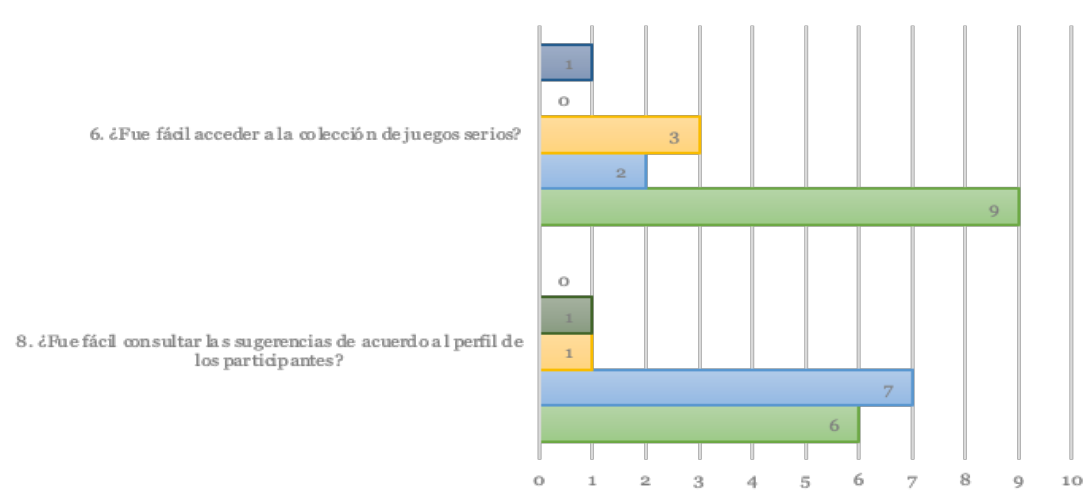

口Totalment e en desacuerdo $\square$ En desacuerdo $\square \mathrm{Ni}$ en desacuerdo ni de acuerdo aDe acuerdo $\square$ Totalment e de acuerdo

Figura 6 - Evaluación de la facilidad de uso de la biblioteca

Como se observa en la figura $73.33 \%$ de los expertos están de acuerdo que es fácil acceder a la colección de los juegos serios y el $86.67 \%$ no tuvieron problemas para visualizar las sugerencias. Estos resultados arrojan que todavía se pueden mejorar las instrucciones o guías de uso de la biblioteca.

La Figura 7 muestra los resultados a las preguntas 7 y 9 que evalúan la presentación de las características de la biblioteca. 


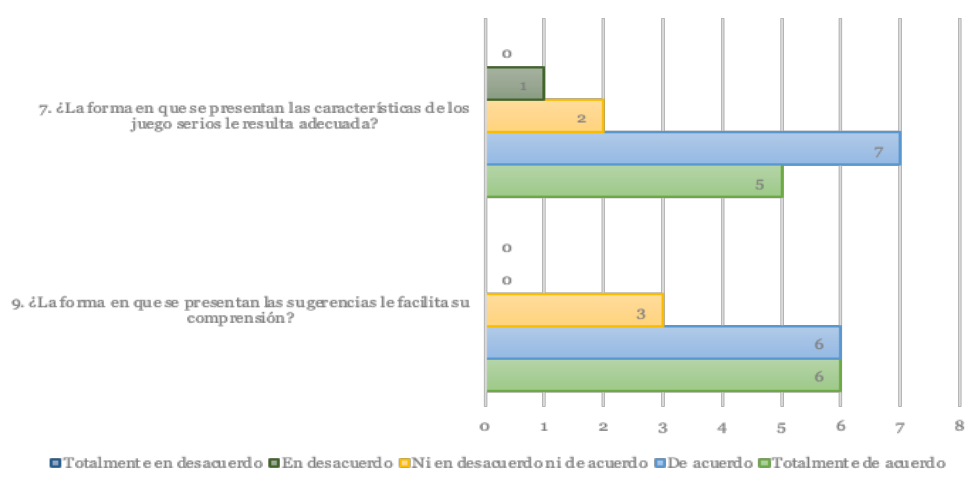

Figura 7 - Evaluación de la presentación de la biblioteca

Como se oberva en la figura, 80\% de los expertos coincidieron estar de acuerdo que la forma en la que se presentan las características de los juegos serios y las sugerencias es adecuada. Nuevamente estos resultados demuestran que es posible mejorar la presentación de los elementos de la biblioteca.

\section{Conclusiones}

Kanban, que fue adaptado a la Ingeniería de Software, ha ganado popularidad tanto dentro como fuera de la industria del software, sin embargo, esta popularidad puede tener sus riesgos, ya que implementarlo erróneamente puede dar resultados esperados. Por ello, la importancia de aplicar métodos de capacitación robustos que enseñen los conceptos básicos de Kanban y cómo implementarlo eficientemente.

Este artículo presenta una propuesta que tiene como propósito ser un repositorio concentrado de juegos serios para la enseñanza de Kanban, como apoyo para los entrenadores y profesores con poca o nula experiencia para facilitar la elección de aquellos que puedan ser de mayor utilidad con base a objetivos de aprendizaje establecidos.

La biblioteca fue validada por expertos en Kanban que evaluaron tres características de la biblioteca: utilidad, presentación y facilidad de uso. Con base en los resultados obtenidos, se puede concluir que la biblioteca es útil como herramienta de apoyo para entrenadores y profesores junior que enseñan Kanban.

La biblioteca, por lo tanto, brinda una solución al problema de hacer efectiva la enseñanza en Kanban, sin embargo, aún falta mucho trabajo por hacer, como la creación de un instrumento de evaluación que permita saber que juegos son los más adecuados para enseñar objetivos específocos de Kanban, además de incrementar el catálogo de juegos serios. 


\section{Referencias}

Agendashift. (2015). Featureban. Recuperado de https://www.agendashift.com/ resources/featureban

Agile42. (2020). Kanban Pizza Game. Recuperado de https://www.agile42.com/en/ agile-teams/kanban-pizza-game/

Ahmad M. O., Markkula, J., \& Oivo, M. (2013). Kanban in Software Development: A Systematic Literature Review. https://doi.org/10.1109/SEAA.2013.28

Anderson, D. J, \& Carmichael, A. (2016). Essential Kanban Condensed. Lean Kanban University Press. https://books.google.com.mx/books?id=I4HeswEACAAJ

Anderson, D. J. (2010). Kanban: Successful Evolutionary Change For Your Technology Business. Blue Hole Press. https://doi.org/10.1093/infdis/jit779

Burrows, M., \& Hohmann, L. (2014). Kanban from the Inside. Blue Hole Press. https://books.google.com.mx/books?id=bIrnoQEACAAJ

Emmerich, K., \& Bockholt, M. (2016). Serious Games Evaluation: Processes, Models, and Concepts. In R. Dörner, S. Göbel, M. Kickmeier-Rust, M. Masuch, \& K. Zweig (Eds.), Entertainment Computing and Serious Games: International GI-Dagstuhl Seminar 15283, Dagstuhl Castle, Germany, July 5-10, 2015, Revised Selected Papers (pp. 265-283). Springer International Publishing. https://doi.org/10.1007/978-3319-46152-6_11

GetKanban. (2020). GetKanban. Recuperado de https://getkanban.com/

Ghannem, A. (2014). Characterization of Serious Games Guided by the Educational Objectives. Proceedings of the Second International Conference on Technological Ecosystems for Enhancing Multiculturality, 227-233. https://doi.org/10.1145/2669711.2669904

Heikkilä, V. T., Paasivaara, M., \& Lassenius, C. (2016). Teaching university students Kanban with a collaborative board game. 471-480. https://doi.org/10.1145/2889160.2889201

Hernández, M., \& Cadavid, J. (2019). A Systematic Literature Review on Organizational Training Using Game-Based Learning. In: 4th Iberoamerican Workshop, HCICollab 2018, Popayán, Colombia, April 23-27, 2018, Revised Selected Papers (pp. 1-18). https://doi.org/10.1007/978-3-030-05270-6_1

IBM Research. (2014). Software Development. IBM Recuperado de: https://researcher. watson.ibm.com/researcher/view_group.php?id=5227

Jagger, J. (2021). Jon Jaggers' Kanban 1s Board Game. Recuperado de https://vdocuments.mx/jon-jaggers-kanban-1s-board-game.html

Larson, K. (2020). Serious Games and Gamification in the Corporate Training Environment: a Literature Review. TechTrends, 64(2), 319-328. https://doi.org/10.1007/s11528-019-00446-7 
Leopold K. (2021). Paper Boats Flow Simulation - Facilitation Package. Recuperado de https://www.squirrelnorth.com/post/paper-boats-flow-simulation-facilitationpackage

Liker, J. K., Hoseus, M., \& Center for Quality People and Organizations. (2008). Toyota Culture: The Heart and Soul of the Toyota Way. McGraw-Hill Education. https://books.google.com.mx/books?id=mfqDBQLlinkC

LitheSpeed. (2020). An exercise in flow - The Dice Game. Recuperado de https://lithespeed.com/an-exercise-in-flow-the-dice-game/

Lopez, F., \& Petrillo, F. (2017). SimKan: Training Kanban Practices Through Stochastic Simulation. https://doi.org/10.1007/978-3-319-55907-0_10

Michael, D., \& Chen, S. (2006). Serious Games: Games That Educate, Train, and Inform.

Netmind. (2020). El juego ágil "Penny Game": descubre la influencia de los tickets en un tablero Kanban. Recuperado de https://netmind.net/es/el-juego-agil-pennygame/

Ohno, T. (1988). Toyota Production System: Beyond Large-Scale Production. Taylor \& Francis. https://books.google.com.mx/books?id=QebEDwAAQBAJ

Salesforce. (2020). Heroku. Recuperado de https://www.heroku.com

State of Agile (2019). 13th Annual State of Agile Report. CollabNet VersionOne, 13, 16. Recuperado de: https://stateofagile.com/\#ufh-i-613553418-13th-annual-state-ofagile-report/7027494

Susi, T., Johannesson, M., \& Backlund, P. (2015). Serious Games - An Overview.

Tastycupcakes (2020). Kanban Tetris. Recuperado de https://www.tastycupcakes. org/2012/o8/kanban-tetris/

Tastycupcakes (2020). Decoration Kanban. Recuperado de https://www.tastycupcakes. org/2017/o7/decoration-kanban/

Unbound DNA (2014). Sudokuban a Kanban in action - Puzzle Game. Recuperado de http://www.unbounddna.com/resources/agile-games/sudokuban-a-kanban-inaction-puzzle-game/

Wovchko, B. (2016). Ending stale stand-ups with Ask Kanban. HUGE I/O Adventures in Lean \& Agile. Recuperado de https://blog.huge.io/ending-stale-stand-ups-withask-kanban-64de6co84d6o

Zyda, M. (2005) From visual simulation to virtual reality to games. Computer, 38(9), 25-32 